\title{
Konik Işınlı Bilgisayarlı Tomografi ile Maksiller Sinüs Septanın Değerlendirilmesi: Retrospektif Klinik Çalışma
}

\author{
Assessment of Maxillary Sinus Septa Using Cone Beam \\ Computed Tomography: A Retrospective Clinical Study
}

Ali Kılınç ${ }^{1}$,

Dilek Menziletoğlu ${ }^{1}$,

Bozkurt Kubilay Işık ${ }^{1}$

${ }^{1}$ Necmettin Erbakan Üniversitesi, Diş

Hekimliği Fakültesi, Konya, Türkiye

Geliş Tarihi/Received: 14 Nisan 2019

Kabul Tarihi/Accepted: 7 Ağustos 2019

Yazışma Adresi: Ali Kılınç, Necmettin Erbakan Üniversitesi, Diş Hekimliği Fakültesi, Konya, Türkiye

e-mail: dtalikilinc@gmail.com

\section{ORCID}

Ali Kılınç

https://orcid.org/0000-0002-2270-6931

Dilek Menziletoğlu

https://orcid.org/0000-0002-2539-0688

Bozkurt Kubilay Işık

https://orcid.org/0000-0002-5724-9400

\begin{abstract}
Öz
Amaç: Bu çalışma maksiller sinüs septa prevalansını, yüksekliğini, anatomik dağılımını dişli ve dişsiz maksillalarda konik ışınlı bilgisayarlı tomografi kullanarak değerlendirmek için yapılmıştır.

Hastalar ve Yöntem: Bu tanımlayıcı çalışma Haziran 2016 ve Şubat 2018 tarihleri arasında 661 hastadan elde edilen 1322 maksiller sinüs konik ışınlı bilgisayarlı tomografi görüntülerinin retrospektif olarak değerlendirilmesi ile yapıldı. Çalışmada 1322 maksiller sinüsün 1092'si dişli hastalardan, 230'u dişsiz hastalardan oluşmaktaydı.

Bulgular: 661 hastanın (373 kadın, 288 erkek) yaş ortalaması $42.1 \pm 18.2$ idi. 1322 maksiller sinüste ortalama yüksekliği $7.95 \pm 4.03 \mathrm{~mm}$ olan toplam 468 (\% 35.4) septa kaydedilmiştir. 468 sinüs septasının çoğunluğu maksiller sinüsün arka bölgesinde idi. Bunlardan 121'i (\% 25.8) ön, 140'ı (\% 29.9) orta ve 207'si (\% 44.2) arka bölgede idi. Maksiller sinüs septa dağılımı dişli ve dişsiz çenelerde değerlendirildiğinde ise 387 dişli hastada (\% 35.4 ) ve 81 dişsiz hastada (\% 35.2) septa varlığı mevcuttu.

Sonuç: Maksiller sinüsün tüm bölümlerinde çeşitli yükseklik ve şekillerde septa görülme sıklığı yüksektir. Bu nedenle sinüs cerrahisi sırasında olası komplikasyonları önlemek için uygun bir radyografik teknikle kapsamlı değerlendirme yapılmalıdır.
\end{abstract}

Anahtar Kelimeler: Maksiller Sinüs, Septa, Konik Işınlı Bilgisayarlı Tomografi

Abstract

Aim: The present study was undertaken to evaluate the prevalence, height and location of maxillary sinus septa in patients with a dentate and an edentulous maxilla using cone beam computed tomography imaging.

Patients and Methods: This descriptive study was conducted on a retrospective evaluation of cone beam computed tomography images of 1322 maxillary sinus with 661 subjects from January 2016 to February 2018. The study consisted of 1322 maxillary sinuses: 1092 from patients with a dentate and 230 from patients with an edentulous maxilla.

Results: The mean age of the 661 patients (373 women, 288 men) was $42.1 \pm 18.2$ years. A total of $468(35.4 \%)$ septa was recorded in 1322 maxillary sinuses with a mean height was $7.95 \pm 4.03 \mathrm{~mm}$. Of 468 sinus septa, the majority was located in the posterior region of the maxillary sinus. Of these, 121 $(25.8 \%)$ were in the anterior, $140(29.9 \%)$ were in the middle and $207(44.2 \%)$ were in the posterior region. Regarding the status of the dentition in the maxilla in relation to the distribution of sinus septa, septa were present in $387(35.4 \%)$ dentate and in $81(35.2 \%)$ edentulous regions.

Conclusion: The prevalence of septa is high in all parts of the maxillary sinus. For this reason, comprehensive evaluation should be performed with an appropriate radiographic technique to prevent possible complications during sinus surgery.

Key words: Maxillar Sinus, Septa, Cone Beam Computed Tomography
Atıf yapmak için: Kılınç A, Menziletoğlu D, Işık BK. Konik Işınlı Bilgisayarlı Tomografi ile Maksiller Sinüs Septanın Değerlendirilmesi: Retrospektif Klinik Çalışma. Selcuk Med J 2020; 36(3): 173-177

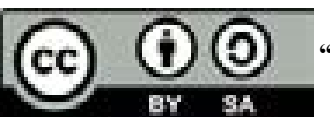

Açıklama: Yazarların hiçbiri, bu makalede bahsedilen herhangi bir ürün, aygıt veya ilaç ile ilgili maddi çıkar ilişkisine sahip değildir. Araştırma, herhangi bir dış organizasyon tarafından desteklenmedi. Yazarlar çalışmanın birincil verilerine tam erişim izni vermek ve derginin talep ettiği takdirde verileri incelemesine izin vermeyi kabul etmektedirler. 


\section{GíRiş}

Maksiller sinüs, 1651 'de Highmore (1) tarafından tanımlanmış ve "Highmore boşluğu" olarak da adlandırımıştır (2). Maksiller sinüs; maksillanın orbital, alveolar, fasiyal ve infratemporal yüzeyleri arasında kalan ince duvarlı büyük bir piramidal kavitedir. Sinüsün büyüklüğü, şekli ve duvar kalınlığı, kişiden kişiye göre değişmektedir ve hatta aynı bireyin iki tarafındaki sinüsler de birbirinden farklı olabilir (3). Sinüs anterior yönde genellikle kanin-premolar dişler bölgesine kadar uzanır. Konveks şekilli olan sinüs tabanı en derin noktasına çoğunlukla birinci molar diş bölgesinde ulaşır (4). Maksiller sinüs septa, ilk olarak 1910'da Underwood (5) tarafından tanımlanmıştır. Maksiller sinüsün içinde, alt veya lateral duvarlarında ters bir gotik ark şeklindeki kortikal kemik duvarlarıdır. Sinüsü iki veya daha fazla boşluğa bölebilir (6). Krennmair ve ark.(7) septaları, primer ve sekonder septa olarak sınıflandırmışlardır. Primer septa maksillanın gelişimi ile meydana gelirken, sekonder septa ise diş kaybından sonra sinüs tabanının düzensiz pnömatizasyonu ile oluşur (8).

Maksiller sinüste yapılacak sinüs tabanı yükseltme operasyonu veya endoskopik sinüs cerrahisi gibi cerrahi girişimlerde maksiller sinüsün anatomisinin ve anatomik varyasyonlarının bilinmesi gerekmektedir. Bu durum cerrahi işlemin doğru bir şekilde planlanmasına ve olası komplikasyonların önlenmesine olanak sağlamaktadır $(9,10)$. Bu çalışmanın amacı; maksiller sinüs septa prevalansını, yüksekliğini, anatomik dağılımını dişli ve dişsiz hastalarda konik ışınlı bilgisayarlı tomografi (KIBT) kullanarak değerlendirmektir.

\section{HASTALAR VE YÖNTEM}

Bu retrospektif çalışma, Ocak 2016 - Şubat 2018 tarihleri arasında Necmettin Erbakan Üniversitesi, Diş Hekimliği Fakültesi, Ağız, Diş ve Çene Cerrahisi bölümüne başvuran 661 hastanın 1322 maksiller sinüsünün KIBT görüntülerinin retrospektif olarak değerlendirilmesiyle yapılmıştır. Necmettin Erbakan Üniversitesi Diş Hekimliği Fakültesinden etik kurul onayı alınıp incelemeler yapılmıştır (2019/02). Bu çalışmada incelenen 1322 maksiller sinüsün 1092'si dişli maksillaya, 230'u ise dişsiz maksillaya sahip olan hastalara aitti. Dental implantlar, endodontik işlemler, oral ve periodontal cerrahi, ortodontik işlemler ve patolojik durumları incelemek için elde edilen tomografiler çalışmaya dahil edilmiştir. Artifaktlı, greftlenmiş ve sinüs patolojisi olan tomografi görüntüleri çalışma dışı bırakılmıştır. KIBT taramaları

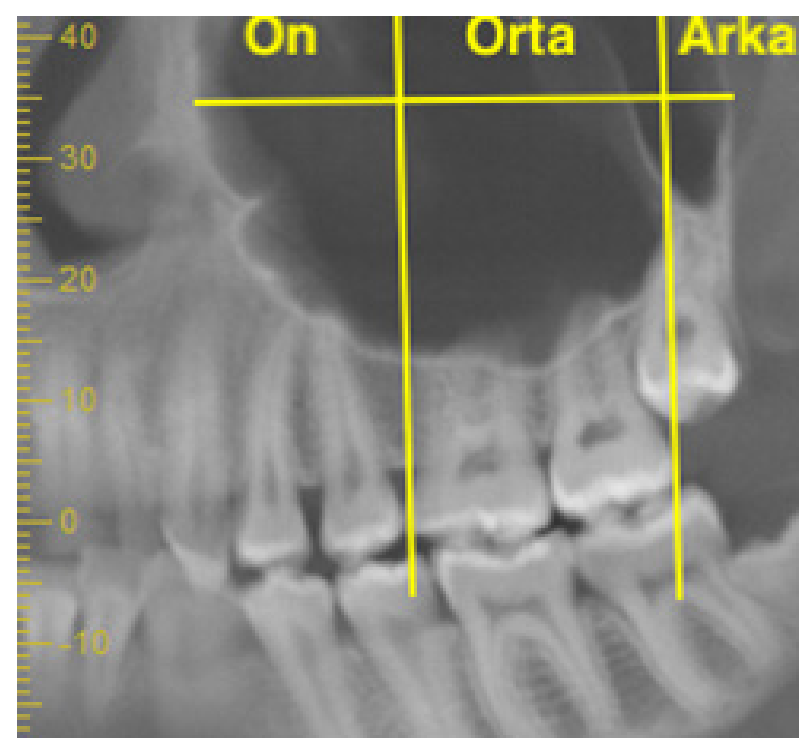

Şekil 1. Dişli hastalarda septa lokalizasyonunu belirlemek için kullanılan yöntem

üzerinde septanın varlığı ve sayısı, yüksekliği, konumu gibi özellikler tüm hastalarda sağ ve sol maksiller sinüsler için ayrı ayrı kaydedildi. KIBT görüntüleri 3D Accuitomo 170 machine (Morita, Kyoto, Japan) cihaz kullanılarak elde edilmiştir.

Septaların konumlarını daha doğru tanımlamak için, sinüsler Kim (2006) ve ark.'nın (11) dişli hastalarda uyguladıkları protokole göre; ön (ikinci premolar dişin distal kısmının daha mezialinde), orta (ikinci premolar dişin distal kısmı ile ikinci molar dişin distal kısmı arasında) ve arka (ikinci molar dişin distal kısmının daha distalinde) olmak üzere üç anatomik alana bölünmüştür. (Şekil 1) Diş eksikliği nedeniyle referans olarak kullanılacak nokta bulunmadığı durumlarda septa lokalizasyonu belirlemek için GonzálezSantana ve ark. ile Rancitelli ve ark.'nın $(10,12)$ tarif ettiği metod kullanıldı. Bu tekniğe göre tomografiden elde edilen panoramik görüntüde sinüsün anterior duvarı ile posterior duvarı arasındaki maksimum mesafe ölçüldü. Bu mesafenin 1/2'si orta, kalan 1/4'lük kısımları ise ön ve arka bölgeler olmak üzere sinüs septlarının lokasyonlarını belirlemek için kullanıldı. (Şekil 2)

Septa yüksekliğinin ölçümü için septanın yaklaşık olarak tabanında olacak şekilde çizilen bir çizgi (AB) çekildi; ve bu çizgiden septa en koronal kısmına kadar uzanan bir çizgi (C-D) kullanılarak da yükseklik ölçümü yapıldı (Şekil 3). Eğer septanın yüksekliği 


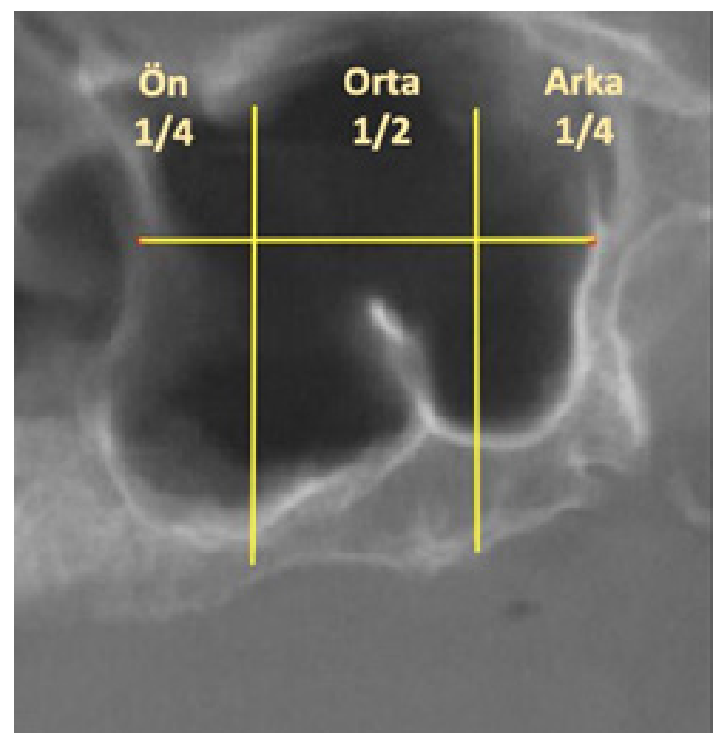

Şekil 2. Dişsiz hastalarda septa lokalizasyonunu belirlemek için kullanılan yöntem

2.5 mm'nin üzerinde ise çalışmaya dahil edildi $(12,13)$. Tüm septa tanımlamaları ve ölçümleri tek bir araştırmacı tarafından yapıldı.

\section{BULGULAR}

Çalışmaya dahil edilen 661 hastanın (373 kadın,

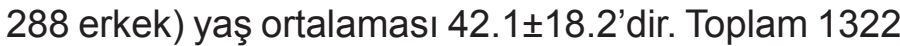
maksiller sinüsün 468 'inde (\% 35.4) septa mevcuttu

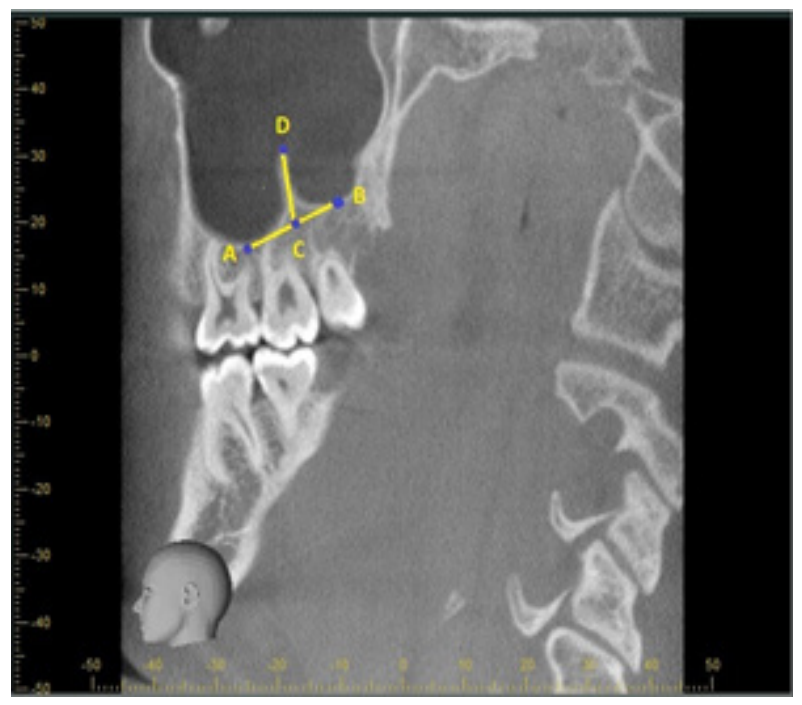

Şekil 3. Septaların ölçüm tekniği ve ortalama septa yüksekliği ise $7.95 \pm 4.03 \mathrm{~mm}$ olarak bulundu. 468 sinüs septanın büyük çoğunluğu maksiller sinüsün arka bölgesinde idi. Bu septaların 121 'i (\% 25.8) ön, 140'ı (\% 29.9) orta ve 207'si (\% 44.2) arka bölgede idi. Maksiller sinüs septa varlığı dişli ve dişsiz çenelerde değerlendirildiğinde ise dişli hastaların \% 35.4'ünde septa mevcut iken ( $n=387)$, dişsiz hastaların ise \%35.2'sinde $(n=81)$ septa varlığı tespit edilmiştir.

\section{TARTIŞMA}

Dental implantlar, kısmi ve total dişsizliği fonksiyonel olarak restore etmek için kullanılan yöntemler arasındadır. Dental implant tedavisi, konvansiyonel sabit ve hareketli protezlere hem konservatif hem de estetik bir alternatif sunan kanıtlanmış ve güvenli bir tedavi yöntemidir. Fakat cerrahi bölgede mevcut kemik yetersizse implant tedavisi mümkün olmayabilir. Bundan dolayı öncelikle implant için gerekli olan kemiği sağlamak gerekmektedir.

Dişsiz posterior maksillada sinüsün genişlemesine bağlı olarak alveolar kemiğin hacmindeki ve vertikal yüksekliğindeki değişimler göz önüne alındığında, implant yerleştirmek için bu alandaki kemiğin hacmini ve boyutunu arttırmak gerekmektedir (14). Posterior maksillada implant tedavisi, dişsiz bölgede maksiller sinüsün pnömatizasyonu nedeniyle daha karmaşıktır. Dişsiz posterior maksiller alveoler krette kemik yüksekliği rezorpsiyona bağlı olarak zamanla azalmaktadır. Bu durum, maksiller sinüsün pnömatizasyonuyla daha da kötüleşir (15). Alveoler kretin kemik yüksekliği kişiden kişiye değişmektedir $(16,17)$. Bu nedenle bazı kişilerde implant tedavisi için vertikal kemik yüksekliği az olabilmektedir. $\mathrm{Bu}$ durumda yeterli kemik oluşturmak için sinüs yükseltme işlemi yaygın olarak kullanılmaktadır. Sinüs yükseltme prosedürü ilk kez 1976'da, atrofik posterior maksillaya implant yerleştirilmesi için Tatum tarafından sözlü olarak tarif edilmiş fakat ilk olarak lateral sinüs duvarında giriş penceresi oluşturup, Schneiderian membranın yükseltilmesi sonrası kemik grefti ile doldurularak yapılan lateral yaklaşım tekniği ile sinüs yükseltme işlemi 1980 yılında Boyne ve James tarafından yayımlanmıştır. (18). Sonraki birkaç yıl içinde de bu cerrahi tekniğin bazı modifikasyonları olmuştur $(19,20)$.

Sinüs tabanı yükseltme işlemi için çeşitli komplikasyonlar bildirilmiştir (21-23) En sık karşılaşılan komplikasyon Schneiderian membranın perforasyonudur $(22,24,25)$. Membran kalınlığı, sinüslerin şekli ve sinüs septa varlığı Schneiderian 
membran perforasyonunu etkileyen faktörlerdir (2628). Özellikle maksiller sinüs tabanındaki septa varlığı, dental implant yerleştirilebilmesi için sinüs yükselmesi işlemi sırasında sinüs membranının perforasyon riskini arttırır $(4,8)$.

Maksiller sinüs septa etiyolojisini araştıran çalışmalar mevcuttur. $(5,29,30)$. Underwood (5), septanın dişlerin farklı sürme evreleri nedeniyle oluştuğunu bildirirken, Neivert (30) septanın etmoid infundibulumun embriyolojik çıkıntısından köken alan parmak benzeri çıkıntılardan meydana geldiğini öne sürmektedir. Ayrıca Krennmair ve ark.(7) septaları, primer ve sekonder septa olarak sınıflandırmışlardır. Primer septa maksillanın gelişimi ile meydana gelirken, sekonder septa ise diş kaybından sonra sinüs tabanının düzensiz pnömatizasyonu ile oluşur (8). Maksiller sinüs septasını tanımlamak için dental panoramik radyografi, bilgisayarlı tomografi (BT) ve konik ışınlı bilgisayarlı tomografi (KIBT) kullanılmaktadır $(4,31,32)$. Uzun yıllar boyunca, cerrahi işlem öncesinde septaların radyografik olarak tanımlanması için panoramik radyografiler kullanılmıştır. Panoramik radyografiler septa gibi anatomik oluşumların belirlenmesinde net ve kesin bir yöntem değildir; fakat bu yöntem hala preoperatif sinüs değerlendirmesinde teşhis için sık kullanılan bir tekniktir (33). Krennmair ve ark. panoramik radyografinin, vakaların \% 21.3'ünde antral septanın pozitif veya negatif olarak tanımlanması konusunda yanlış tanıya yol açabileceğini bildirmiştir (7).

Maksiller sinüs septa varlığı atrofik arklarda sinüs yükseltme işlemi sırasında birtakım zorluklar ortaya koymaktadır. Bundan dolayı, herhangi bir cerrahi prosedür başlatılmadan önce maksiller sinüs septanın varlığının bilinmesi son derece önemlidir. $\mathrm{Bu}$ sayede cerrahlar Schneiderian membranının perforasyonu gibi komplikasyonları önlemek için cerrahi tekniklerini değiştirebilirler ve başka önlemler alabilirler (2). Sinüs sayısı esas alınarak yapılan çalışmalarda, maksiller sinüs septa prevalansı \% 13 ile \% 35.3 arasında değişmektedir. Hasta sayısı esas alınarak yapılan çalışmalarda ise prevalans $\% 21.6$ ile \% 66.7 arasında değişmektedir (6). Yıldırım ve ark. 1000 maksiller sinüsü KIBT üzerinde inceledikleri çalışmada \%29.7 oranında septa prevalansı tespit etmişlerdir (32). Bu oranlardaki farklılıkların sebebi çalışmalardaki görüntüleme yöntemlerinin farklı olmasından kaynaklandığı düşünülmektedir. Çünkü bazı çalışmalar septa varlığını tespit ederken panoramik radyografi üzerinden bazı çalışmalar ise KIBT üzerinden değerlendirmiştir. Çalışmamızdaki septa prevalansı \% 35.4 olarak bulunmuştur. Dişli hastalar ve dişsiz hastalardaki septa prevalansı bazı çalışmalarda farklı bulunurken (33) bazı çalışmalarda benzer bulunmaktadır (32). Çalışmamızda dişli hastalarda septa prevalansı \% 35.4 iken dişsiz hastalarda \% 35.2 olarak hesaplanmıştır. Dişli ve dişsiz hastalarda septa varlığı açısından farklı bulunmamıştır.

Septanın anatomik dağılımı, Kim ve ark. (11), 100 hastada yaptıkları çalışmada ön bölgede $\% 25$, orta bölgede \% 51 ve arka bölgede \% 24 olarak bildirmiştir. Lee ve ark. (34) ise çalışmalarında septa dağılımını \% 27.3 oranında ön, \% 50 oranında orta ve \%22.7 oranında arka bölgede bulmuşlardır. Çalışmamızda ise ön bölgede \% 25.8 orta bölgede \% 29.9 ve arka bölgede \% 44.2 oranında bulunmuştur. Literatüre bakıldığında septa yükseklikleri arasında farklı ortalamalar da görülmektedir. Gosau ve ark. (35) çalışmalarında septa yüksekliği ortalamasını $5.4 \mathrm{~mm}$, Rosano ve ark. (36) ise $8.72 \mathrm{~mm}$ olarak bildirmişlerdir. Ulm ve ark. (13) yaptıkları çalışmalarında da çalışmamıza benzer şekilde septa yükseklik ortalamasını $7.9 \mathrm{~mm}$ olarak bildirmişlerdir. Çalışmamızda septa yüksekliği ortalaması $7.95 \pm 4.03 \mathrm{~mm}$ olarak kaydedilmiştir. Yükseklik ortalamalarının farklı olması, çalışma gruplarının farklı kökenden olmasından, ölçüm için kullanılan tekniklerin ve görüntüleme yöntemlerinin farklılığından ve ayrıca dahil etme kriterlerinin farklı olmasından kaynaklanabileceğini öngörmekteyiz.

Sonuç olarak; Maksiller sinüsün tüm bölümlerinde çeşitli yükseklik ve şekillerde septa görülme sıklığı yüksektir. Dişli ve dişsiz hastalarda septa prevalansı açısından fark yoktur. Sinüs cerrahisi sırasında olası komplikasyonları önlemek için uygun bir radyografik teknikle kapsamlı değerlendirme yapılmalıdır.

Çıkar Çatışması: Çalışmada herhangi bir çıkar çatışması yoktur.

Finansal Çıkar Çatışması: Çalışmada herhangi bir finansal çıkar çatışması yoktur.

Yazışma Adresi: Ali Kılınç, Necmettin Erbakan Üniversitesi, Diş Hekimliği Fakültesi, Konya, Türkiye e-mail:dtalikilinc@gmail.com

\section{KAYNAKLAR}

1. Maestre-Ferrín L, Galán-Gil S, et al. Maxillary sinus septa: A systematic review. Med Oral Patol Oral Cir Bucal 2010;15:383-6.

2. Bhattacharya PT, Patil K, Guledgud MV. Maxillary sinus septa: A panoramic radiographic study. Int J Stomatol Occlusion Med 2015;8:92-6.

3. Arman C, Ergür I, Atabey A, et al. The thickness and the 
lengths of the anterior wall of adult maxilla of the West Anatolian Turkish people. Surg Radiol Anat 2006;28:553-8.

4. Koymen R, Gocmen-Mas N, Karacayli U, et al. Anatomic evaluation of maxillary sinus septa: Surgery and radiology. Clin Anat 2009;22:563-70.

5. Underwood AS. An inquiry into the anatomy and pathology of the maxillary sinus. J Anat Physiol 1910;44:354-69.

6. Maestre-Ferrín L, Galán-Gil S, Rubio-Serrano M, et al. Maxillary sinus septa: A systematic review. Med Oral Patol Oral Cir Bucal 2010;15:e383-6.

7. Krennmair G, Ulm CW, Lugmayr $\mathrm{H}$, et al. The incidence, location, and height of maxillary sinus septa in the edentulous and dentate maxilla. J Oral Maxillofac Surg 1999;57:667-71; discussion 671-2.

8. Gülşen U, Mehdiyev İ, Üngör C, et al. Horizontal maxillary sinus septa: An uncommon entity. Int J Surg Case Rep 2015;12:67-70.

9. Orhan K, Kusakci Seker B, Aksoy S, et al. Cone beam CT evaluation of maxillary sinus septa prevalence, height, location and morphology in children and an adult population. Med Princ Pract 2013;22:47-53.

10. González-Santana H, Peñarrocha-Diago M, Guarinos-Carbó $\mathrm{J}$, et al. A study of the septa in the maxillary sinuses and the subantral alveolar processes in 30 patients. J Oral Implantol 2007;33:340-3.

11. Kim M-J, Jung U-W, Kim C-S, et al. Maxillary sinus septa: Prevalence, height, location, and morphology. A reformatted computed tomography scan analysis. J Periodontol 2006;77:903-8.

12. Rancitelli $D$, Borgonovo $A E$, Cicciù $M$, et al. Maxillary sinus septa and anatomic correlation with the schneiderian membrane. J Craniofac Surg 2015;26:1394-8.

13. Ulm CW, Solar P, Krennmair G, et al. Incidence and suggested surgical management of septa in sinus-lift procedures. Int $\mathrm{J}$ Oral Maxillofac Implants 1995;10:462-5.

14. Taleghani F, Tehranchi M, Shahab S, et al. Prevalence, location, and size of maxillary sinus septa: Computed tomography scan analysis. J Contemp Dent Pract 2017;18:115 .

15. Natarajarathinam G, Rao A, Kannaperuman J, et al. Crosssectional study estimating prevalence of maxillary sinus septum in South Indian population. Journal of Dental Implants 2015;5:16.

16. Cawood JI, Howell RA. A classification of the edentulous jaws. Int J Oral Maxillofac Surg 1988;17:232-6.

17. Vinter I, Krmpotić-Nemanić J, Hat J, et al. [Does the alveolar process of the maxilla always disappear after tooth loss?]. Laryngorhinootologie 1993;72:605-7.
18. Boyne PJ, James RA. Grafting of the maxillary sinus floor with autogenous marrow and bone. J Oral Surg 1980;38:6136.

19. Farmand M. Horse-shoe sandwich osteotomy of the edentulous maxilla as a preprosthetic procedure. J Maxillofac Surg 1986;14:238-44.

20. Sailer HF. A new method of inserting endosseous implants in totally atrophic maxillae. Journal of Cranio-Maxillofacial Surgery 1989;17:299-305.

21. Beaumont C, Zafiropoulos G-G, Rohmann K, et al. Prevalence of maxillary sinus disease and abnormalities in patients scheduled for sinus lift procedures. J Periodontol 2005;76:461-7.

22. Kasabah S, Slezák R, Simůnek $A$, et al. Evaluation of the accuracy of panoramic radiograph in the definition of maxillary sinus septa. Acta Medica 2002;45:173-5.

23. Maksoud MA. Complications after maxillary sinus augmentation: A case report. Implant Dent 2001;10:168-71.

24. Schwartz-Arad D, Herzberg R, Dolev E. The prevalence of surgical complications of the sinus graft procedure and their impact on implant survival. J Periodontol 2004;75:511-6.

25. Chan $\mathrm{H}-\mathrm{L}$, Wang $\mathrm{H}-\mathrm{L}$. Sinus pathology and anatomy in relation to complications in lateral window sinus augmentation. Implant Dent 2011;20:406-12.

26. Decision tree to minimize intraoperative complications during maxillary sinus augmentation procedures. Journal of Oral Biology 2018;5:1-8.

27. Wen S-C, Lin Y-H, Yang Y-C, et al. The influence of sinus membrane thickness upon membrane perforation during transcrestal sinus lift procedure. Clin Oral Implants Res 2015;26:1158-64.

28. Wen S-C, Chan H-L, Wang H-L. Classification and management of antral septa for maxillary sinus augmentation. Int J Periodontics Restorative Dent 2013;33:509-17.

29. Stover JD. The incidence, location, and height of maxillary sinus septa in the edentulous and dentate maxilla. J Oral Maxillofac Surg 1999;57:671-2.

30. Neivert $H$. Surgical anatomy of the maxillary sınus. Laryngoscope 1930;40:14.

31. Bornstein MM, Scarfe WC, Vaughn VM, et al. Cone beam computed tomography in implant dentistry: A systematic review focusing on guidelines, indications, and radiation dose risks. Int J Oral Maxillofac Implants 2014;29 Suppl:55-77.

32. Talo Yildirim T, Güncü G-N, Colak M, et al. Evaluation of maxillary sinus septa: A retrospective clinical study with cone beam computerized tomography (CBCT). Eur Rev Med Pharmacol Sci 2017;21:5306-14. 